\title{
BMJ Open Impact of early life nutrition on gut health in children: a prospective clinical study
}

\author{
Delphine Ley, ${ }^{1}$ Laurent Beghin, ${ }^{1,2}$ Jules Morcel, ${ }^{1,2}$ Florence Flamein, ${ }^{2}$ \\ Charles Garabedian, ${ }^{3,4}$ Bertrand Accart, ${ }^{5}$ Elodie Drumez, ${ }^{4,6}$ Julien Labreuche, ${ }^{4,6}$ \\ Frederic Gottrand, ${ }^{1,2}$ Emmanuel Hermann (D) ${ }^{1}$
}

To cite: Ley D, Beghin L, Morcel J, et al. Impact of early life nutrition on gut health in children: a prospective clinical study. BMJ Open 2021;0:e050432. doi:10.1136/ bmjopen-2021-050432

- Prepublication history for this paper is available online. To view these files, please visit the journal online (http://dx.doi org/10.1136/bmjopen-2021050432).

Received 26 February 2021 Accepted 03 August 2021

\section{Check for updates}

(c) Author(s) (or their employer(s)) 2021. Re-use permitted under CC BY-NC. No commercial re-use. See rights and permissions. Published by BMJ.

For numbered affiliations see end of article.

\section{Correspondence to} Dr Emmanuel Hermann; emmanuel.hermann@univlille.fr

\section{ABSTRACT}

Introduction The first 1000 days of life could contribute to individual susceptibility to the later development of chronic non-communicable diseases. Nutrition in early life appears to be an important determinant factor for a sustainable child's health. In this study, we propose to investigate the impact of exclusive breast feeding on gut health in children.

Methods and analysis A prospective cohort of newborns $(n=350)$ will be recruited at birth and followed up to 4 years of age. The main objective is to evaluate the link between exclusive breast feeding for at least 3 months and the gut health of the child at 4 years. The primary endpoint of assessment of gut health will be based on the non-invasive measurement of faecal secretory $\lg A$ (slgA) as a sensitive biomarker of the intestinal ecosystem. The presence of gastrointestinal disorders will be defined according to the clinical criteria of Rome IV. Information on parent's nutritional habits and life style, breastfeeding duration and child's complementary feeding will be collected along the follow-up. Cord blood cells and plasma at birth will be purified for further analysis. The meconium and stools collected at birth, 6 months, 2 years and 4 years of age will allow slgA analysis.

Ethics and dissemination This clinical study has obtained the approval from the national ethical committee. We plan to publish the results of the study in peer-review journals and by means of national and international conference.

Trial registration number NCT04195425.

\section{INTRODUCTION}

The first 1000 days extending from conception to the end of the first 2 years of life define a period of intense development and growth of the fetus and infant. A growing body of evidence supports the central role of perinatal environment in programming individual predisposition to chronic non-communicable diseases. ${ }^{1-5}$ Many studies strongly suggest that perinatal factors such as mode of delivery, type of infant feeding or antibiotic therapy during the first months of life influence the later risk of chronic intestinal disease. ${ }^{46}$ However, the links between nutrition during

\section{Strengths and limitations of this study}

- This is a prospective and longitudinal mother/child cohort with minimal constraints (3 visits over 4 years) and minimal risk (no intervention and minimally invasive procedures)

- The study will collect a great deal of longitudinal information on children during the first years of life, their parents and their environment via questionnaires and biological samples (cord blood, meconium, stools).

- This study will obtain precise data on breastfeeding practices and their short-term and medium-term effects on the health of the child, in particular and in an innovative way on gut health through stool samples for immunological analysis, and by using the Rome IV paediatric questionnaire.

- Weaknesses could be relied to confounding factors at the selection process and during the follow-up. The nutritional survey during the study is primarily based on reports from parents and is retrospective which could contribute to inaccuracies due to long delay between each visit.

- Due to the non-interventional study design, we will be able to study association but no causal relationship.

the first 1000 days, gut development and lifelong health remain to be better understood.

Breast feeding is the reference for infant feeding who should be exclusively breastfed for at least the first 6 months for optimal growth. ${ }^{7}$ The influence of breast feeding on short-term health and infant development has been demonstrated. In particular, breast feeding is associated with superior cognitive development, decreased risk of ear, respiratory and gastrointestinal infections and atopic diseases. ${ }^{8}$ The consensus is much weaker with regard to longer-term beneficial effects of breast feeding on outcome of chronic non-communicable diseases. ${ }^{8-14}$ Human milk contains all of the essential nutrients as well as bioactive factors that are 
thought to contribute to the health benefits of breastfed infants. ${ }^{15} 16$ Among these bioactive factors, secretory IgA (sIgA), human milk oligosaccharides, lactoferrin and milk fat globule membrane, have demonstrated role in shaping intestinal barrier function, immune development and composition of the intestinal microbiota, suggesting that breastmilk may have beneficial effect in programming long-term gut health. ${ }^{5}{ }^{617-20}$ The understanding of imprint of early life nutrition on gut homoeostasis is a concern for the development of preventive strategies aimed at promoting gut health.

We designed a prospective clinical study 'Périnatalité, Environnement, Santé Intestinale et Nutrition de l'Enfant' (PENSINE) to evaluate the impact of exclusive breast feeding on gut health in children beyond 6 months of age and to identify the underlying mechanisms involved. The cohort of newborns $(n=350)$ will be recruited at birth and followed up to 4 years of age. The nutritional habits and life style of parents, breastfeeding duration and complementary feeding of the child will be surveyed. Cord blood cells and meconium will be harvested at birth and stools will be collected at 6 months, 2 years and 4 years of age for implementing a biobanking. Gut health will be based on faecal sIgA as the primary study outcome parameter. sIgA is known to regulate gut inflammation, intestinal microbiota richness and protection against gastrointestinal infections. ${ }^{21-23}$ Its level in gut is under the influence of breast feeding in infants but scarce data are available in toddler. ${ }^{10}$ Immune and microbiome analysis will be coupled to the evaluation of the presence of gastrointestinal disorders by using the Rome IV paediatric questionnaire. $^{2425}$

\section{Study objective}

The overall objective of the study PENSINE is to get more insight into the association between infant's nutritional environment and gut health. The follow-up will focus beyond the duration of the '1000 days for Health' until the age of 4 years. This work should strengthen our initial hypothesis that early determinants such as breast feeding has sustainable influence on gut health in preschool children.

\section{Primary objective}

To evaluate the link between exclusive breast feeding for at least 3 months (this threshold was chosen because beyond 3 months of age the frequency of exclusive breast feeding is low in France ${ }^{26}$ ) and gut health at 4 years of age. Gut health will be evaluated based on the non-invasive measurement of faecal sIgA as a sensitive biomarker of the intestinal ecosystem. ${ }^{1027-29}$ Besides the measurement of $\operatorname{sIgA}$ as the primary outcome parameter, the presence of gastrointestinal disorders will be assessed using the Rome IV criteria. ${ }^{24}$ Biological samples collected from birth to 4 years of age will allow to carry out a biobanking. The stool collection will further permit the analysis of other inflammatory markers and metagenomic study of the intestinal microbiota.

\section{Secondary objectives}

To evaluate the association between gut health (sIgA) at 4 years of age and the duration of exclusive breast feeding.

To describe the mother's eating habits during pregnancy (Food Frequency Questionnaire) and their links with gut health during the first 4 years of life.

To assess the association between the level of sIgA in stool during the first 4 years of life with the child's growth and the presence of gastrointestinal disorders.

\section{METHODS AND ANALYSIS}

\section{Study population and setting}

The study is carried out at the Jeanne de Flandre University Hospital in Lille in the Northern France. This region is known to have a significant proportion of patients with chronic non-communicable diseases including inflammatory bowel diseases (IBDs). However, the frequency of functional gastrointestinal disorders in infants and preschool children is unknown. Although the initiation of breast feeding in the Northern France was observed to be weak in $2016(54 \%),{ }^{30}$ in the Jeanne de Flandre maternity it is estimated that more than $70 \%$ of mothers initiate breast feeding during their stay in the hospital (personal communication).

In our study, 350 mother and newborn dyads will be included. The maternity enjoys around 5600 births a year with a recruitment capacity fully satisfactory for our study. When the eligible mother's birth intention will be recorded at the Jeanne de Flandre maternity (Lille University Hospital) they will be fully informed during the third trimester of pregnancy by the investigator with an information letter detailing the content and the relevance of the study. The inclusion of the pregnant women and future fathers will be consecutive and carried out during the third trimester of pregnancy after the signing of the informed consent by each of the parents. The inclusion of the child will take place after birth. Both parents will sign a second specific informed consent for the child's participation.

The inclusion criteria will be as follows: parents $\geq 18$ years old when signing the consent form; the ability to speak, read and write French, or being able to be assisted in completing questionnaires; pregnant mothers living in the Lille urban area; single pregnancy. Mothers who will be unable to participate in the study follow-up, that is, mother under curatorship/guardianship or who participate in another clinical study incompatible with biological collection, will be excluded from the study. The exclusion criteria a posteriori for the child after birth will be: prematurity ( $<37$ weeks of gestation), gastrointestinal congenital malformation, gastrointestinal surgery during the first 6 months of life and inability to participate in the study follow-up. Child will be also excluded from the study if they are under justice protection measure or if they participate in another clinical study incompatible with biological collection. 
Pregnant mothers will be included in the study whether or not initiating breast feeding. Exclusive breast feeding will be defined as no intake of other liquids than breastfed (water, sugar water, herbal tea, fruit juice, prebiotics or probiotics supplementation, with the exception of oral rehydration solution or vitamins and medicines) as stated by WHO. ${ }^{31}$ Six months of exclusive breast feeding should be the optimal way of infant feeding ${ }^{7}$ but this remains scarce in France. ${ }^{26}$ Among mothers who start breast feeding at maternity, mixed breast feeding will be considered when the child receives breast milk and infant formula, or other liquids. All children will be scheduled for three clinical visits, at 6 months, 2 years and 4 years of age. Since the time between each visit is long, the study needs to ensure a reminder for the family to take care about the study. Therefore, a semiannual newsletter will be sent to the families concerned by the study. The bulletin will include information on the progress of the PENSINE study as well as an educational section on the first 1000 days and gut health. Families will be contacted between 1 and 2 months before each visit to make sure all is well and make an appointment.

Since December 2020, the date of the first inclusion, more than 90 inclusions of parents have been made to date. We plan to complete the inclusions in 2022 and the full study in 2026. The study will last 5 years. All administrative and regulatory issues will be managed by the Lille University Hospital Health Research Directorate including data protection and ethical. The logistical coordination of this project will be under the responsibility of the paediatric Clinical Investigation Centre
(CIC-1403-Inserm-CHU) of the Lille University Hospital involved in clinical data handling and biobanking.

\section{Study design}

It will be a prospective clinical study including 350 mother/child dyads recruited at the Lille University Jeanne de Flandre maternity (figure 1). The follow-up of the children will be carried out from birth until the age of 4 years. The diagram of the research process is divided into three periods (figure 2): prenatal period (period 1: (inclusion of the pregnant mother, study of the nutritional profile and lifestyle of the pregnant woman), birth of the child (period 2: inclusion of the child and collection of perinatal factors), followed by 4 years (period 3: clinical and nutritional follow-up of the child).

1. Prenatal period (1): During the third trimester of pregnancy, mothers will have to filled out a questionnaire on their socioeconomic level and a Questions of Food Frequency questionnaire relating to their eating habits, providing information on their consumption of meat and fish, fruit or vegetables, dairy products, fat or sugar, industrial products. ${ }^{32}$ Health information of the mother and medication received including antibiotics during pregnancy, as well as gastrointestinal history of parents, will be collected from the obstetric medical record.

2. Birth period (2): Birth information, including mode of delivery and per partum antibiotic, will be collected from the mother's obstetrical record. Cord blood will be taken to store cord blood mononuclear cells (CBMC) and fetal plasma as part of a biobanking. Chil-

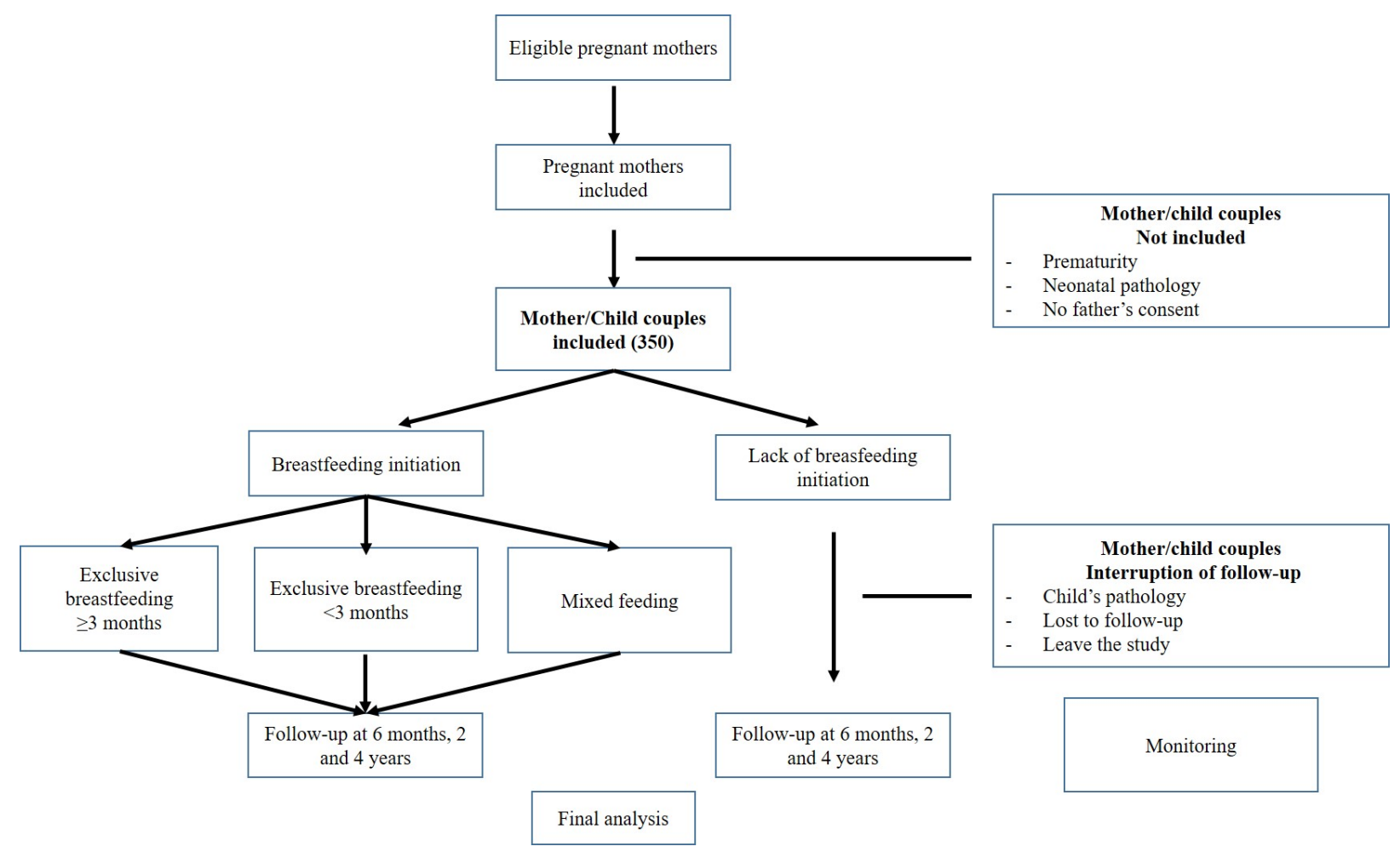

Figure 1 Study design. 


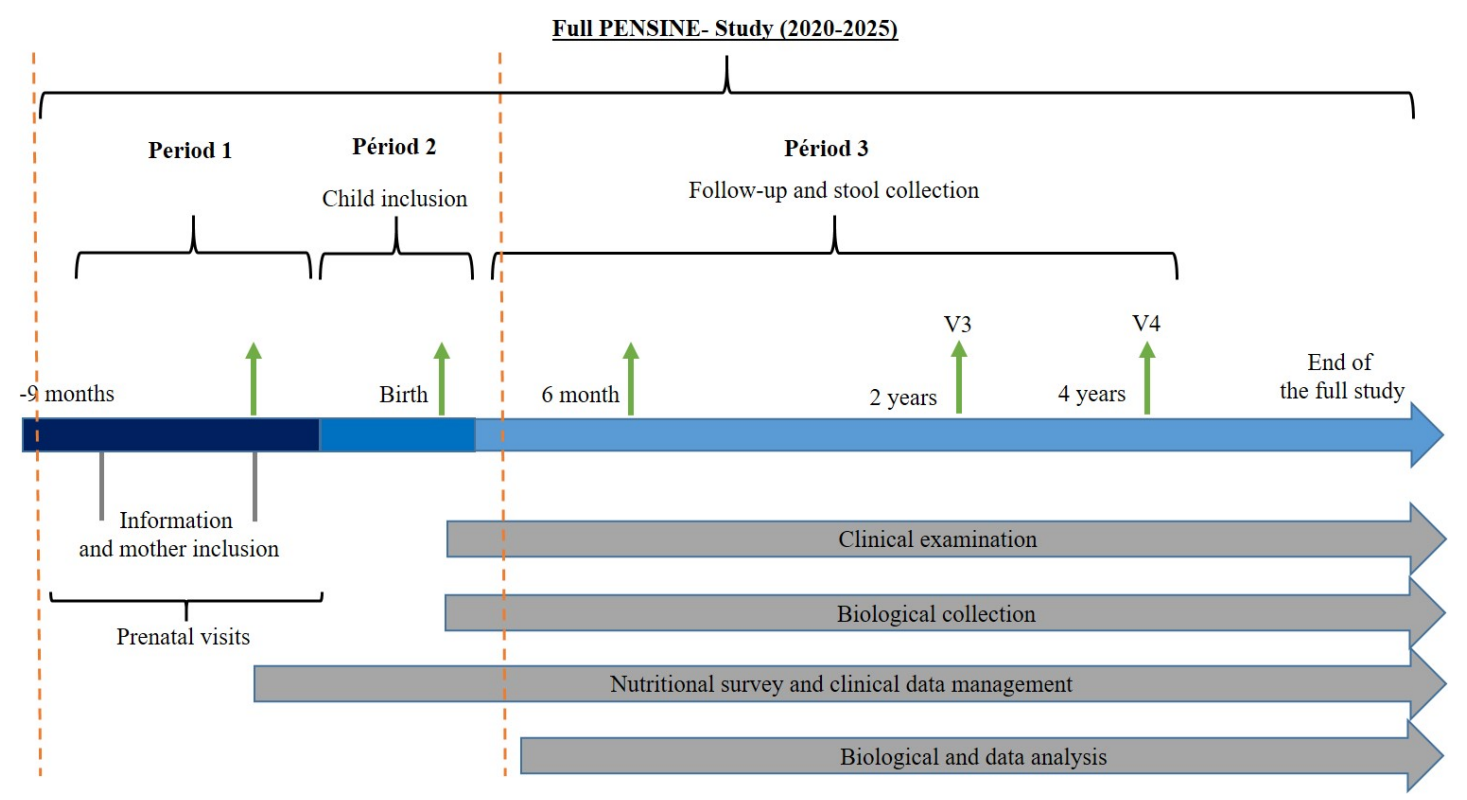

Figure 2 Study schedules. PENSINE, Périnatalité, Environnement, Santé Intestinale et Nutrition de l'Enfant.

dren's meconium will be collected to be frozen and stored.

3. Follow-up period (3) after birth: Three follow-up visits will be scheduled at $6 \pm 1$ months, $24 \pm 1$ months and $48 \pm 1$ months at the paediatric CIC. The visit will include: clinical examination of the child, anthropometric measurements, collection of medical information including episodes of fever, diarrhoea or atopic dermatitis and medication received, standardised food questionnaires (Food and life style question, see table 1), and questionnaires intended to diagnose the presence of functional gastrointestinal disorders in children (Rome IV). The Rome IV criteria are validated, symptoms-based criteria for the diagnosis of functional gastrointestinal disorders in infants and children, including regurgitations, colic, constipation and diarrhoea. The presence of these functional gastrointestinal disorders will be determined at each follow-up visit using a standardised validated questionnaire based on the Rome IV criteria. ${ }^{2433}$ The stools of infants will be collected at each time, aliquot and stored for further immunological studies (faecal sIgA and inflammatory markers such

\section{Table 1 Objectives of PENSINE surveys}

\section{Questionnaires \\ Socioeconomic questionnaire \\ Food Frequency Questionnaires for mothers during pregnancy}

Food and life style questionnaires at 6 months of age

\section{Aims}

To describe the socioeconomic level

To describe food profiles ('meat eaters'/'vegetable eaters', Mediterranean diet, western diet, etc...)

To describe the duration of exclusive breast feeding (main criterion of comparison between two groups: breastfed or not breastfed).

To evaluate infant milk intake (infant formula vs breast feeding).

To describe the timing of food diversification.

To describe the quality of food diversification.

To evaluate the type of care (child alone, in a 'small group' or in a group).

Food and life style questionnaire at 2 years of age To evaluate the duration of breast feeding, beyond the 6 months.

To evaluate infant milk intake.

To describe several food profiles ('meat eaters'/'vegetable eaters'

Mediterranean diet, Western diet, etc...).

To evaluate the mode of care.

Food and life style questionnaire at 4 years of age To describe several food profiles ('meat eaters'/'vegetable eaters'

Mediterranean diet, Western diet, etc...).

To evaluate the mode of care.

Rome IV questionnaire at 6 months, 2 years and 4 To describe gastrointestinal disorders in the child.

years of age 
as faecal calprotectin) and metagenomic analysis of the intestinal microbiota.

\section{Patient and public involvement}

Parents included in this study will not be involved in the design, recruitment or conduction of the study.

\section{Sample size}

The study was powered to demonstrate an effect size (standardised mean difference) in sIgA at 4 years $\geq 0.40$ considered as medium. This effect size was chosen given the effect size of exclusive breast feeding on gut health measured by sIgA at 3 months of life reported in literature. ${ }^{10}$ To demonstrate this effect size at 4 years, assuming an exclusive breastfeeding rate of $40 \%$ at 3-month of age, with a two-sided test at $5 \%$ of significance and statistical power of $80 \%, 208$ subjects (mother/child dyad) are needed. To take into account $40 \%$ of subjects who cannot be analysed at 4 years, 350 subjects should be included. Because premature infants are excluded, the inclusion of pregnant mothers in the third trimester of pregnancy will take place until the goal of the inclusion of 350 eligible full-term newborns at birth is reached. Considering $10 \%$ prematurity, we anticipate the inclusion of 390 pregnant mothers.

\section{Data management}

The study will provide a set of medical and dietary outcomes for each participant during pregnancy, at birth and during the follow-up. The individual data collected during the study will be reported on a source document and then will be recorded into a computer database (electronic-case report form (e-CRF)). The processing of data will be carried out under the conditions of confidentiality defined by the French law of 6 January 1978 modified relating to data processing, files and freedoms (Commission Nationale de l'Informatique et des Libertés) as well as in accordance with European Regulation (General Data Protection Regulation). Access to the data will be restricted to those directly involved in the study.

The data concerning this study will be archived for a minimum period of fifteen years from the end of the research or its early termination without prejudice to the legislative and regulatory provisions in force.

\section{Medical and anthropometric data}

Medical data will be recorded at baseline by trained staff. They will include medications as well as complications (hypertension, pre-eclampsia, gestational diabetes, thromboembolic complications, anaemia, prolonged rupture of membranes) during pregnancy or labour and/or childbirth, mode of delivery, birth weight, length and head circumference of the newborn. These data will be considered for the final analysis. Anthropometric parameters will be measured as part of clinical assessment at birth, 6 months, 2 years and 4 years of age in infants and children with standardised techniques. Information on the exposure of relevant drugs will be recorded at baseline and during the study (using standardised questionnaires). The presence of gastrointestinal disorders will be assessed by a paediatric gastroenterologist using the paediatric Rome IV diagnostic criteria ${ }^{33}$ and recorded in the e-CRF.

\section{Biobanking}

All samples will be kept frozen at the Biological Resource Centre (BRC) of the Lille University Hospital to constitute a biobank until the analysis. The BRC/CIC will ensure the management of biological samples with a view to their subsequent analysis, storage and management of these in its aspects of referencing, storage, traceability of inputs, outputs or incidents.

According to the primary outcome, an aliquot of stool from 4-year-old children will be used to measure sIgA by ELISA methodology (commercial kit). The other biological elements of the biobank (cord blood plasma, CBMC, meconium, aliquots of stool) will be available as part of ancillary research projects by our team or open to the scientific and clinical community. These research projects might include analysis of immunological biomarkers, composition of the intestinal microbiota on stool samples and epigenetics on CBMC.

\section{Blood samples}

After delivery, cord blood will be drawn in heparinised tubes by the midwife from the umbilical vein after clamping and before cutting the cord. The labelled tube will be transferred to the BRC for processing. Plasma and purified CBMCs will be frozen and stored as part of the protocol. After collecting and distributing the plasma in an aliquot, CBMCs will be purified and cryopreserved in liquid nitrogen for biobanking.

At the age of 4 years, a finger prick will be proposed to collect a drop of blood of the child using a lancing device. The drop of blood will be placed on a Whatman filter paper using the dried blood spot technique for subsequent analysis of inflammatory serological biomarkers.

\section{Fecal samples}

At birth, meconium will be collected under sterile conditions by midwives or paediatric nurses, then transferred to the BRC for aliquoting and freezing. At 6 months, 24 months and 48 months, the child's stool will be collected by the parents at home in the days before the visit. Faecal collection kits prepared by the BRC will be used. Stools will be placed in a labelled sterile container before storing at $+4^{\circ} \mathrm{C}$. The stool sample will be brought back to the CIC for the follow-up visit. The sample will be immediately transferred to the $\mathrm{BRC}$ in order to aliquot, manage traceability and storage at $-80^{\circ} \mathrm{C}$ for further immunological and intestinal microbiome analysis.

\section{Statistical analysis}

Statistical analysis will be performed by the Methodology, Biostatistics and Data Management Department at the Lille University Hospital under the responsibility of Julien Labreuche and Elodie Drumez. Data will be 
analysed using the SAS software 9.4. (SAS Institute). All statistical tests will be performed with a two-tailed alpha risk of 0.05 ; all secondary objectives will be considered as exploratory and no correction for multiple comparisons will be applied. A detailed statistical analysis plan will be written and finalised prior to the database lock.

Primary objective: the sIgA level assessed at 4-year of life will be compared according to exclusive breastfeeding status at 3-month of age using a Student's t-test; the standardised mean difference will be calculated with its $95 \%$ CI. To account prespecified confounding factors (age of dietary diversification, socioeconomic level of parents, gestational age at birth, mode of delivery, antibiotic therapy during the first months), a multivariable linear regression model will be used, with sIgA level as dependent variable, and exclusive breastfeeding status at 3-month of age, and prespecified confounding factors as independent variables. To account missing data on dependent and independent variables, primary analysis will be performed after handling missing values by multiple imputation procedure under the missing at random assumption using a regression switching approach (chained equation with number of imputation $(\mathrm{m})$ guided by maximal fraction of missing information $/ \mathrm{m}<0.1) .{ }^{34}$ The imputation models will be used all baseline and 3-month characteristics, and study outcome (sIgA level at 4-year of life) with predictive mean matching method for quantitative variables and logistic regression (binary, ordinal or polynomial) for categorical variables). Estimates obtained in the different imputed data sets will be combined using Rubin's rules. ${ }^{35}$ A complete-case analysis will be done as first sensitivity analysis. A second sensitivity analysis will be done by using the Hayden method to account the rate of missing information on primary outcome, by using a weighted linear regression model. ${ }^{36}$ The weights will be calculated as the probabilities of missing outcome value in each group of interest estimated using multivariable logistic regression model including missing information status as dependent variable and all baseline and 3-month characteristics as independent variables.

Secondary objectives: Briefly, to evaluate the association of sIgA at 4 years of age with the duration of exclusive breast feeding (assessed as ordinal 5-level categorical variable) a one-way analysis of variance will be performed; a trend test will be done using linear contrast. To account prespecified confounding factors, a multivariable linear regression model will be performed.

To assess the association of mother's eating habits during pregnancy (measured by Food Frequency Questionnaire) and the gut health during the first 4 years of life (assessed at 6 months, 2 years and 4 years), we will used a linear mixed model (with unstructured covariance matrix to account the repeated measures), by included mother's eating habit characteristics, time and time* mother's eating habit characteristics as fixed effects. Post hoc comparison at each time of follow-up will be done using linear contrast. As specified above, multivariate analysis will be done by considering the same prespecified confounding factors into linear mixed model. We also used the same approach to assess the association of child's growth and the presence of gastrointestinal disorders and the level of sIgA in stool first 4 years of life (assessed at 6 months, 2 years and 4 years). For all linear regression models, the normality of residuals will be checked.

\section{DISCUSSION AND PERSPECTIVES}

Previous studies on the link between total faecal sIgA and breast feeding were restricted to the first months of life,

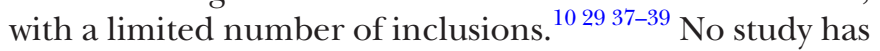
investigated the relationships between neonatal nutrition, faecal sIgA, intestinal microbiota and gastrointestinal disorders during the first years of life. In our study, gastrointestinal disorders will be diagnosed for the first time with the paediatric Rome IV questionnaire.

The gastrointestinal tract undergoes significant maturation during the perinatal period, which leads to the establishment of the intestinal barrier and immune homoeostasis. The production of sIgA detectable in stools is higher at 6 months of age in breastfed infants compared with those receiving infant formula. ${ }^{10}{ }^{37}$ sIgA plays a key role in programming intestinal homoeostasis and is a relevant biomarker of gut health. ${ }^{21}{ }^{28}{ }^{29}$ Moreover, the composition of the intestinal microbiota differs significantly between breastfed infants and those receiving infant formula (higher proportion of bifidobacteria and lactobacilli which are overall beneficial for health in breastfed infants). ${ }^{5}$ The cessation of breast feeding, more than the introduction of weaning foods, is the main driver in the dynamics of microbiota development during the first year of life. ${ }^{40}$ On the other hand, the impact of the weaning stage on microbiota development has been considerably less investigated, but is thought to contribute to intestinal microbiota alpha-diversity. ${ }^{5}$

The gut microbiome has been shown to be highly involved in many critical physiological processes, including food digestion, immune system development and metabolic homoeostasis. A growing number of studies have reported that early changes in human gut microbiota diversity may affect risk factors for chronic diseases such as IBD, allergy or diabetes. ${ }^{45}$ Microbes are believed to colonise the neonatal gut immediately following birth and shape host immunity. ${ }^{5}$ Although the microbiota in children seems to reach an adult-like diversity around 2-3 years of age, some recent investigations claimed the structure of gut microbial community is still developing in preschool children. ${ }^{42}$

These data clearly underscore the importance of better understanding how breast milk affects gut health in infancy. The PENSINE study will allow the child's health trajectory to be understood as a function of gut health, its clinical impacts during infancy and later on in preschool children (focused on 4 years), and the complex intricacies between multiple factors including the perinatal environmental exposure, nutritional status, inflammation, microbiota or epigenetic modifications. The data 
from the complete study will provide a conceptual basis for thinking about the possibility of nutritional recommendations for mothers and children with regard to a sustainable health trajectory. The biobank will also open up transversal perspectives for clinical research with a significant impact on clinicians in the field of neonatology, obstetrics, paediatrics and nutrition.

This study encompasses a number of strength and limitations. This is a prospective and longitudinal mother/child cohort with minimal constraints (3 visits over 4 years) and minimal risk (no intervention and minimally invasive procedures). It will collect a great deal of longitudinal information on children during the first years of life, their parents and their environment via questionnaires and biological samples (cord blood, meconium, stools). This study will obtain precise data on breastfeeding practices and their short-term and mediumterm effects on the health of the child, in particular and in an innovative way on gut health through stool samples for immunological analysis, and by using the Rome IV paediatric questionnaire. Weaknesses could be relied to confounding factors at the selection process and during the follow-up. By addition, the nutritional survey during the study is primarily based on reports from parents and is retrospective which could contribute to inaccuracies due to long delay between each visit. Finally, due to the non-interventional study design we will be able to study association but no causal relationship.

\section{Ethics and dissemination}

The clinical study was approved by the French national ethical committee (CPP Sud-Est VI ClermontFerrand/2019-A01416-51/18 October 2019). It will be conducted in accordance with the clinical study protocol, and with the Good Clinical Practices. In particular, the study participant (legal representative of the child) will receive fair and complete information through the information letter, explained orally by the investigator or the midwife. Each legal representative of the child will give their written consent on a consent form prior to fill out the questionnaires and to collect biological samples.

The first deliverables of the study will consist in establishing regular reports, and at the end of the study a final report will be written by the medical investigator. This vast study will lead to clinical and biological results that will be communicated through publications and communications by poster or oral at specialist congresses or seminars. Our study is totally in the field of early health programming, which is the main theme of the Developmental Origins of Health and Disease, which organises conferences in which many elements of our study might be presented. In conclusion, the identification of risk profiles for intestinal diseases and the rapid introduction of new recommendations to improve health outcomes will be major outlets for this study. Collectively, the overall project will produce appropriate knowledge useful for regulatory and risk management agencies and even for related stakeholders in order to support specific recommendations.

\section{Author affiliations}

${ }^{1}$ Univ. Lille, Inserm, CHU Lille, U1286 - INFINITE - Institute for Translational Research in Inflammation, F-59000 Lille, France

${ }^{2}$ Univ. Lille, Inserm, CHU Lille, CIC-1403 Inserm-CHU, F-59000 Lille, France

${ }^{3}$ Univ. Lille, CHU Lille, Department of Obstetrics \& Gynecology, F-59000 Lille, France ${ }^{4}$ Univ. Lille, CHU Lille, ULR 2694-METRICS: évaluation des technologies de santé et des pratiques médicales, F-59000 Lille, France

${ }^{5} \mathrm{CHU}$ Lille - Inserm - UL, CRB/CIC1403, F-59037 Lille, France

${ }^{6} \mathrm{CHU}$ Lille, Department of Biostatistics, F-59000 Lille, France

Acknowledgements We thanks all people who contributed in the development of the protocol. We thank the biological resource centre for biobanking.

Contributors DL, LB and EH designed the project and drafted the manuscript. FG supervised the project. JM contributes to the inclusion and reviewed the manuscript, FF, CG, BA, ED and JL reviewed the manuscript. All authors read and approved the final version of the manuscript.

Funding This work was supported by Fondation Roquette Pour la Santé (placed under the aegis of Fondation de France (grant 00092409)), GIRCI (Groupement Interrégional de Recherche Clinique et d'Innovation) Nord Ouest (grant AAP-AE18-14) and FHU (Fédération Hospitalo-Universitaire) '1000 days for health' (grant AAP-2019 - FHU 1000 jours).

\section{Competing interests None declared.}

Patient and public involvement Patients and/or the public were not involved in the design, or conduct, or reporting, or dissemination plans of this research.

Patient consent for publication Not required.

Provenance and peer review Not commissioned; externally peer reviewed.

Open access This is an open access article distributed in accordance with the Creative Commons Attribution Non Commercial (CC BY-NC 4.0) license, which permits others to distribute, remix, adapt, build upon this work non-commercially, and license their derivative works on different terms, provided the original work is properly cited, appropriate credit is given, any changes made indicated, and the use is non-commercial. See: http://creativecommons.org/licenses/by-nc/4.0/.

\section{ORCID iD}

Emmanuel Hermann http://orcid.org/0000-0003-0971-4918

\section{REFERENCES}

1 Roseboom T, de Rooij S, Painter R. The Dutch famine and its longterm consequences for adult health. Early Hum Dev 2006;82:485-91.

2 Barker DJ, Winter PD, Osmond C, et al. Weight in infancy and death from ischaemic heart disease. Lancet 1989;2:577-80.

3 Indrio F, Martini S, Francavilla R, et al. Epigenetic matters: the link between early nutrition, microbiome, and long-term health development. Front Pediatr 2017;5:178.

4 Ley D, Desseyn J-L, Mischke M, et al. Early-Life origin of intestinal inflammatory disorders. Nutr Rev 2017;75:175-87.

5 Milani C, Duranti S, Bottacini F, et al. The first microbial colonizers of the human gut: composition, activities, and health implications of the infant gut microbiota. Microbiol Mol Biol Rev 2017;81. doi:10.1128/ MMBR.00036-17. [Epub ahead of print: 0811 2017].

6 Ley D, Desseyn J-L, Gouyer V, et al. Early life nutrition influences susceptibility to chronic inflammatory colitis in later life. Sci Rep 2019;9:18111.

7 The World Health Organization. The optimal duration of exclusive breastfeeding. Report of an expert consultation. WHO/NHD/0109 2001.

8 Victora CG, Bahl R, Barros AJD, et al. Breastfeeding in the 21st century: epidemiology, mechanisms, and lifelong effect. Lancet 2016;387:475-90.

9 Rozé J-C, Darmaun D, Boquien C-Y, et al. The apparent breastfeeding paradox in very preterm infants: relationship between breast feeding, early weight gain and neurodevelopment based on results from two cohorts, EPIPAGE and lift. BMJ Open 2012;2:e000834.

10 Bridgman SL, Konya T, Azad MB, et al. Infant gut immunity: a preliminary study of IgA associations with breastfeeding. J Dev Orig Health Dis 2016;7:68-72. 
11 Martin RM, Patel R, Kramer MS, et al. Effects of promoting longer-term and exclusive breastfeeding on adiposity and insulinlike growth factor-I at age 11.5 years: a randomized trial. JAMA 2013;309:1005-13.

12 Gale C, Logan KM, Santhakumaran S, et al. Effect of breastfeeding compared with formula feeding on infant body composition: a systematic review and meta-analysis. Am J Clin Nutr 2012;95:656-69.

13 Differding MK, Doyon M, Bouchard L, et al. Potential interaction between timing of infant complementary feeding and breastfeeding duration in determination of early childhood gut microbiota composition and BMI. Pediatr Obes 2020;15:e12642.

14 Heshmati J, Sepidarkish M, Shidfar F, et al. Effect of breastfeeding in early life on cardiorespiratory and physical fitness: a systematic review and meta-analysis. Breastfeed Med 2018;13:248-58.

15 Boudry G, Charton E, Le Huerou-Luron L I, et al. The relationship between breast milk components and the infant gut microbiota. Front Nutr 2021;8:629740.

16 Thai JD, Gregory KE. Bioactive factors in human breast milk attenuate intestinal inflammation during early life. Nutrients 2020;12. doi:10.3390/nu12020581. [Epub ahead of print: 23 Feb 2020].

17 Parigi SM, Eldh M, Larssen P, et al. Breast milk and solid food shaping intestinal immunity. Front Immunol 2015;6:415.

18 Martin CR, Ling P-R, Blackburn GL. Review of infant feeding: key features of breast milk and infant formula. Nutrients 2016;8. doi:10.3390/nu8050279. [Epub ahead of print: 11 May 2016].

19 Walker WA, lyengar RS. Breast milk, microbiota, and intestinal immune homeostasis. Pediatr Res 2015;77:220-8.

20 Carr LE, Virmani MD, Rosa F, et al. Role of human milk bioactives on infants' gut and immune health. Front Immunol 2021;12:604080.

21 Macpherson AJ, Yilmaz B, Limenitakis JP, et al. Iga function in relation to the intestinal microbiota. Annu Rev Immunol 2018;36:359-81.

22 Moon C, Baldridge MT, Wallace MA, et al. Vertically transmitted faecal IgA levels determine extra-chromosomal phenotypic variation. Nature 2015;521:90-3

23 Rogier EW, Frantz AL, Bruno ME, et al. Lessons from mother: long-term impact of antibodies in breast milk on the gut microbiota and intestinal immune system of breastfed offspring. Gut Microbes 2014:5:663-8.

24 Zeevenhooven J, Koppen IJN, Benninga MA. The new Rome IV criteria for functional gastrointestinal disorders in infants and toddlers. Pediatr Gastroenterol Hepatol Nutr 2017;20:1-13.

25 Campeotto F, Barbaza M-O, Hospital V. Functional gastrointestinal disorders in outpatients aged up to 12 months: a French noninterventional study. Int J Environ Res Public Health 2020;17. doi:10.3390/ijerph17114031. [Epub ahead of print: 0506 2020].

26 Wagner S, Kersuzan C, Gojard S. Breastfeeding duration in France according to parents and birth characteristics. Results from the ELFE longitudinal French study, 2011. Bull Epidemiol Hebd 2015;29:522-32.

27 Béghin L, Tims S, Roelofs M, et al. Fermented infant formula (with Bifidobacterium breve C50 and Streptococcus thermophilus O65) with prebiotic oligosaccharides is safe and modulates the gut microbiota towards a microbiota closer to that of breastfed infants. Clin Nutr 2021;40:778-87.

28 Brandtzaeg P. Secretory lgA: designed for anti-microbial defense. Front Immunol 2013;4:222.

29 Bridgman SL, Konya T, Azad MB, et al. High fecal IgA is associated with reduced Clostridium difficile colonization in infants. Microbes Infect 2016;18:543-9.

30 Blondel B, Gonzalez L, Raynaud P. Enquête nationale périnatale 2016. les naissances et les établissements, situation et évolution depuis 20102016.

31 World Health Organization. Breastfeeding: the World Health Organization, 2021. Available: https://apps.who.int/nutrition/topics/ exclusive breastfeeding/en/index.html

32 Barnaba L, Intorre F, Azzini E, et al. Evaluation of adherence to Mediterranean diet and association with clinical and biological markers in an Italian population. Nutrition 2020;77:110813.

33 Benninga MA, Faure C, Hyman PE, et al. Childhood functional gastrointestinal disorders: Neonate/Toddler. Gastroenterology 2016;150:1443-55. doi:10.1053/j.gastro.2016.02.016

34 Buuren S, Groothuis-Oudshoorn K. Multivariate imputation by Chained equations in R. J Stat Softw 2011;45:1-67.

35 Rubin DB. Multiple imputation for nonresponse in surveys. New York: John Wiley and Sons, 1987.

36 Hayden D, Pauler DK, Schoenfeld D. An estimator for treatment comparisons among survivors in randomized trials. Biometrics 2005;61:305-10.

37 Maruyama K, Hida M, Kohgo T, et al. Changes in salivary and fecal secretory $\lg A$ in infants under different feeding regimens. Pediatr Int 2009:51:342-5.

38 Kukkonen K, Kuitunen M, Haahtela T, et al. High intestinal IgA associates with reduced risk of IgE-associated allergic diseases. Pediatr Allergy Immunol 2010;21:67-73.

39 Viljanen M, Kuitunen M, Haahtela T, et al. Probiotic effects on faecal inflammatory markers and on faecal IgA in food allergic atopic eczema/dermatitis syndrome infants. Pediatr Allergy Immunol 2005;16:65-71.

40 Bäckhed F, Roswall J, Peng Y, et al. Dynamics and stabilization of the human gut microbiome during the first year of life. Cell Host Microbe 2015;17:690-703.

41 Yatsunenko T, Rey FE, Manary MJ, et al. Human gut microbiome viewed across age and geography. Nature 2012;486:222-7.

42 Roswall J, Olsson LM, Kovatcheva-Datchary P, et al. Developmental trajectory of the healthy human gut microbiota during the first 5 years of life. Cell Host Microbe 2021;29:765-76. 